\title{
Numerical Algorithm for Solving General Linear Elliptic Quaternionic Matrix Equations
}

\author{
Kemal Eren $^{1}$ and Hidayet Hüda Kösal ${ }^{1 *}$ \\ ${ }^{1}$ Department of Mathematics, Faculty of Science and Arts, Sakarya University, Sakarya, Turkey \\ * Corresponding author
}

\section{Article Info}

Keywords: Elliptic quaternion, Elliptic quaternion matrix, General linear elliptic quaternionic matrix equations 2010 AMS: $15 A 18,15 B 33$

Received: 1 March 2021

Accepted: 9 September 2021 Available online: 15 September 2021

\begin{abstract}
In this study, we develop a general method to solve the general linear elliptic quaternionic matrix equations by means of real representation of elliptic quaternion matrices. A pseudocode for our approach that provides the solution of the linear elliptic quaternionic matrix equations is expressed. Moreover, we apply this method to the well-known Slyvester matrix equations and Kalman Yakubovich matrix equations over the elliptic quaternion algebra.
\end{abstract}

\section{Introduction and Preliminaries}

Real quaternions are a four-dimensional number system that was first expressed by Hamilton in 1843, based on the idea of generalizing complex numbers [1]. Hamilton first tried to describe the 3-dimensional number system as follows:

$$
q=q_{0}+q_{1} i+q_{2} j
$$

where $q_{0}, q_{1}, q_{2} \in \mathbb{R}$ and $i^{2}=j^{2}=-1$. However, he saw that this number system does not provide the closure property under multiplication. In this way, Hamilton saw that there could not be a system similar to any 3-dimensional complex number system and defined the 4-dimensional number system is known as the real quaternion in the following way:

$$
\mathbb{K}=\left\{q=q_{0}+q_{1} i+q_{2} j+q_{3} k: q_{0}, q_{1}, q_{2}, q_{3} \in \mathbb{R} \text { and } i, j, k \notin \mathbb{R}\right\}
$$

such that

$$
i^{2}=j^{2}=k^{2}=-1, i j=-j i=k, i k=-k i=-j, j k=-k j=i .
$$

There are many applications of real quaternion algebra in different fields of the scientific world. The main areas are kinematics, mechanics, quantum physics, chemistry, image-signal restoration, and game development. For this reason, there are many studies related to real quaternions in literature [2]-[6].

On the other hand, Segre defined commutative quaternions in 1892 [7]. One of the most essential properties of a commutative quaternion is that it meets the commutative property of multiplication. The commutative quaternion algebra is a significant factor in fields such as Hopfield neural networks, digital signal, and image processing [8]-[11]. Therefore, commutative 
quaternion algebra theory has been increasingly important in recent years.

Elliptic quaternions are the generalized form of commutative quaternions. The set of elliptic quaternions is a commutative ring under a commutative law and combination law of a four-dimensional Clifford algebra. Moreover, this set contains non-trivial idempotents, nilpotent elements, and zero-divisors [8, 12, 13].

The set of elliptic quaternions with basic elements $1, i, j$ and $k$ is represented as

$$
\mathbb{H}_{\alpha}=\left\{a=a_{0}+a_{1} i+a_{2} j+a_{3} k: a_{0}, a_{1}, a_{2}, a_{3} \in \mathbb{R} \text { and } i, j, k \notin \mathbb{R}\right\}
$$

which satisfy the equalities $i^{2}=k^{2}=\alpha, j^{2}=1, i j=j i=k, j k=k j=i, k i=i k=\alpha j, \alpha<0, \alpha \in \mathbb{R}$, [8]. Addition of any two elliptic quaternions $a=a_{0}+a_{1} i+a_{2} j+a_{3} k, b=b_{0}+b_{1} i+b_{2} j+b_{3} k \in \mathbb{H}_{\alpha}$ is given by $a+b=\left(a_{0}+b_{0}\right)+$ $\left(a_{1}+b_{1}\right) i+\left(a_{2}+b_{2}\right) j+\left(a_{3}+b_{3}\right) k$. Scalar multiplication of a elliptic quaternion $a \in \mathbb{H}_{\alpha}$ with a scalar $\lambda \in \mathbb{R}$ is expressed as $\lambda a=\lambda\left(a_{0}+a_{1} i+a_{2} j+a_{3} k\right)=\lambda a_{0}+\lambda a_{1} i+\lambda a_{2} j+\lambda a_{3} k$. In addition, the operation of the quaternionic multiplication of two elliptic quaternions $a, b \in \mathbb{H}_{\alpha}$ is expressed as

$$
\begin{aligned}
& a b=\left(a_{0} b_{0}+\alpha a_{1} b_{1}+a_{2} b_{2}+\alpha a_{3} b_{3}\right)+\left(a_{1} b_{0}+a_{0} b_{1}+a_{3} b_{2}+a_{2} b_{3}\right) i \\
& \quad+\left(a_{0} b_{2}+a_{2} b_{0}+\alpha a_{1} b_{3}+\alpha a_{3} b_{1}\right) j+\left(a_{3} b_{0}+a_{0} b_{3}+a_{1} b_{2}+a_{2} b_{1}\right) k
\end{aligned}
$$

On the other hand, we know that the elliptic quaternion $a \in \mathbb{H}_{\alpha}$ has three types of the conjugate: ${ }^{1} \bar{a}=a_{0}-a_{1} i+a_{2} j-a_{3} k,{ }^{2} \bar{a}=$ $a_{0}-a_{1} i-a_{2} j+a_{3} k$ and ${ }^{3} \bar{a}=a_{0}+a_{1} i-a_{2} j-a_{3} k$. Additionally, the norm of the elliptic quaternion $a \in \mathbb{H}_{\alpha}$ is

$$
\|a\|=\sqrt[4]{a\left({ }^{1} \bar{a}\right)\left({ }^{2} \bar{a}\right)\left({ }^{3} \bar{a}\right)}=\sqrt[4]{\left[\left(a_{0}+a_{2}\right)^{2}-\alpha\left(a_{1}+a_{3}\right)^{2}\right]\left[\left(a_{0}-a_{2}\right)^{2}-\alpha\left(a_{1}-a_{3}\right)^{2}\right]} .
$$

If $a \in \mathbb{H}_{\alpha}$ and $\|a\| \neq 0$ then there exists multiplicative inverse of the elliptic quaternion $a$. So, multiplicative inverse of the elliptic quaternion $a$ is $a^{-1}=\frac{\left({ }^{1} \bar{a}\right)\left({ }^{2} \bar{a}\right)\left({ }^{3} \bar{a}\right)}{\|a\|^{4}}[8,12]$.

For

$$
\mathbb{H}_{\alpha}^{\prime}=\left\{\left(\begin{array}{cccc}
a_{0} & \alpha a_{1} & a_{2} & \alpha a_{3} \\
a_{1} & a_{0} & a_{3} & a_{2} \\
a_{2} & \alpha a_{3} & a_{0} & \alpha a_{1} \\
a_{3} & a_{2} & a_{1} & a_{0}
\end{array}\right) \in \mathbb{R}^{4 \times 4}: a_{0}, a_{1}, a_{2}, a_{3} \in \mathbb{R}\right\}
$$

$\mathbb{H}_{\alpha}$ is algebraically isomorphic to the matrix algebra $\mathbb{H}_{\alpha}^{\prime}$ through the bijective map

$$
\phi: \mathbb{H}_{\alpha} \rightarrow \mathbb{H}_{\alpha}^{\prime}, \quad \phi_{a}=\left(\begin{array}{cccc}
a_{0} & \alpha a_{1} & a_{2} & \alpha a_{3} \\
a_{1} & a_{0} & a_{3} & a_{2} \\
a_{2} & \alpha a_{3} & a_{0} & \alpha a_{1} \\
a_{3} & a_{2} & a_{1} & a_{0}
\end{array}\right)
$$

Thus, every elliptic quaternion $a \in \mathbb{H}_{\alpha}$ has a real matrix representation

$$
\phi_{a}=\left(\begin{array}{cccc}
a_{0} & \alpha a_{1} & a_{2} & \alpha a_{3} \\
a_{1} & a_{0} & a_{3} & a_{2} \\
a_{2} & \alpha a_{3} & a_{0} & \alpha a_{1} \\
a_{3} & a_{2} & a_{1} & a_{0}
\end{array}\right)
$$

in $\mathbb{H}_{\alpha}^{\prime}[8]$.

Theorem 1.1. ([8, 12]). For $a, b \in \mathbb{H}_{\alpha}$ and $\lambda \in \mathbb{R}$, the following identities are satisfied:

1. $a=b \Leftrightarrow \phi_{a}=\phi_{b}$,

2. $\phi_{(a+b)}=\phi_{a}+\phi_{b}$,

3. $\phi_{(a b)}=\phi_{a} \phi_{b}$

4. $\phi_{\left(\phi_{(a)} b\right)}=\phi_{a} \phi_{b}$

5. $\phi_{(\lambda a)}=\lambda \phi_{a}$,

6. $\operatorname{trace}\left(\phi_{a}\right)=a+{ }^{1} \bar{a}+{ }^{2} \bar{a}+{ }^{3} \bar{a}$,

7. $\|a\|^{4}=\left|\operatorname{det}\left(\phi_{a}\right)\right|$. 
Let's denote by $\mathbb{H}_{\alpha}^{m \times n}$ which is the set of all $m \times n$ type matrices with elliptic quaternion entries. $\mathbb{H}_{\alpha}^{m \times n}$ with the ordinary matrix summation and multiplication is a ring with identity. The conjugates of elliptic quaternion matrix $A=\left(a_{i j}\right) \in \mathbb{H}_{\alpha}^{m \times n}$ which has three types of conjugate are given the following as:

$$
{ }^{1} \bar{A}=\left({ }^{1} \overline{a_{i j}}\right) \in \mathbb{H}_{\alpha}^{m \times n},{ }^{2} \bar{A}=\left({ }^{2} \overline{a_{i j}}\right) \in \mathbb{H}_{\alpha}^{m \times n} \text { and }{ }^{3} \bar{A}=\left({ }^{3} \overline{a_{i j}}\right) \in \mathbb{H}_{\alpha}^{m \times n}
$$

Also, elliptic quaternion matrix $A=\left(a_{i j}\right) \in \mathbb{H}_{\alpha}^{m \times n}$ can be expressed as $A=A_{0}+A_{1} i+A_{2} j+A_{3} k$ where $A_{0}, A_{1}, A_{2}, A_{3} \in \mathbb{R}^{m \times n}$. Then, ${ }^{1} \bar{A}=A_{0}-A_{1} i+A_{2} j-A_{3} k,{ }^{2} \bar{A}=A_{0}-A_{1} i-A_{2} j+A_{3} k$ and ${ }^{3} \bar{A}=A_{0}+A_{1} i-A_{2} j-A_{3} k$. A matrix $A^{T} \in \mathbb{H}_{\alpha}^{n \times m}$ is transpose of $A \in \mathbb{H}_{\alpha}^{m \times n}$. Also $A^{*}=\left({ }^{s} \bar{A}\right)^{T} \in \mathbb{H}_{\alpha}^{m \times n}, s=1,2,3$, is called conjugate transpose with respect to the $s^{t h}$ conjugate of $A \in \mathbb{H}_{\alpha}^{m \times n}$, [12].

Theorem 1.2. ([12]) Let's assume that A and B are elliptic quaternion matrices of appropriate sizes. Then the following expressions are provided:

1. $\left({ }^{s} \bar{A}\right)^{T}=s \overline{\left(A^{T}\right)}$,

2. $(A B)^{*_{s}}=B^{*_{s}} A^{*_{s}}$,

3. $(A B)^{T}=B^{T} A^{T}$,

4. ${ }^{s} \overline{(A B)}=\left({ }^{s} \bar{A}\right)\left({ }^{s} \bar{B}\right)$,

5. If $A^{-1}$ and $B^{-1}$ exist then $(A B)^{-1}=B^{-1} A^{-1}$,

6. If $A^{-1}$ exists $\left(A^{*_{s}}\right)^{-1}=\left(A^{-1}\right)^{*_{s}}$,

7. $\left({ }^{s} \bar{A}\right)^{-1}=s \overline{\left(A^{-1}\right)}$.

For any elliptic quaternion matrix $A=A_{0}+A_{1} i+A_{2} j+A_{3} k \in \mathbb{H}_{\alpha}^{m \times n}$, the real representation $\Phi_{A}$ of the elliptic quaternion matrix $A$ were given in [13] as follows,

$$
\Phi_{A}=\left(\begin{array}{cccc}
A_{0} & \alpha A_{1} & A_{2} & \alpha A_{3} \\
A_{1} & A_{0} & A_{3} & A_{2} \\
A_{2} & \alpha A_{3} & A_{0} & \alpha A_{1} \\
A_{3} & A_{2} & A_{1} & A_{0}
\end{array}\right) \in \mathbb{R}^{4 m \times 4 n}
$$

in here $A_{0}, A_{1}, A_{2}, A_{3} \in \mathbb{R}^{m \times n}, \alpha \in \mathbb{R}$ and $\alpha<0$.

Theorem 1.3. ([13]) Let $A, B \in \mathbb{H}^{m \times n}, C \in \mathbb{H}_{\alpha}^{n \times p}$ and $\lambda \in \mathbb{R}$ be given. In that case, following identities for the elliptic quaternion matrix are satisfied:

1. $A=B \Leftrightarrow \Phi_{A}=\Phi_{B}, \Phi_{A+B}=\Phi_{A}+\Phi_{B}$,

2. $\Phi_{A C}=\Phi_{A} \Phi_{C}, \Phi_{\lambda A}=\lambda \Phi_{A}$,

3. $A=\frac{1}{2-2 \alpha} E_{4 m} \Phi_{A}\left({ }^{1} \bar{E}_{4 n}\right)^{T}$ where $E_{4 t}=\left(\begin{array}{llll}I_{t} & i I_{t} & j I_{t} & k I_{t}\end{array}\right) \in \mathbb{H}^{t \times 4 t}$,

4. If $A$ is a nonsingular matrix of size $m$, then

$$
\Phi_{A^{-1}}=\Phi_{A}^{-1}, \quad A^{-1}=\frac{1}{2-2 \alpha} E_{4 m} \Phi_{A}^{-1}\left({ }^{1} \bar{E}_{4 n}\right)^{T}
$$

5. $\Phi_{A^{-}}=\Phi_{A}^{-}, A^{-}=\frac{1}{2-2 \alpha} E_{4 m} \Phi_{A}^{-}\left({ }^{1} \bar{E}_{4 n}\right)^{T}$ are generalized inverse of $\Phi_{A}$ and $A$, respectively,

6. $\Phi_{A}=R_{4 m}^{-1} \Phi_{A} R_{4 n}, \Phi_{A}=S_{4 m}^{-1} \Phi_{A} S_{4 n}$ and $\Phi_{A}=T_{4 m}^{-1} \Phi_{A} T_{4 n}$ where

$$
R_{4 t}=\left(\begin{array}{cccc}
0 & \alpha I_{t} & 0 & 0 \\
I_{t} & 0 & 0 & 0 \\
0 & 0 & 0 & \alpha I_{t} \\
0 & 0 & I_{t} & 0
\end{array}\right), S_{4 t}=\left(\begin{array}{cccc}
0 & 0 & I_{t} & 0 \\
0 & 0 & 0 & I_{t} \\
I_{t} & 0 & 0 & 0 \\
0 & I_{t} & 0 & 0
\end{array}\right), T_{4 t}=\left(\begin{array}{cccc}
0 & 0 & 0 & \alpha I_{t} \\
0 & 0 & I_{t} & 0 \\
0 & \alpha I_{t} & 0 & 0 \\
I_{t} & 0 & 0 & 0
\end{array}\right)
$$

\section{On solutions of general linear elliptic quaternionic matrix equations}

In this section, we study the solutions of the equations

$$
A_{1} X B_{1}+\cdots+A_{l} X B_{l}=C
$$

by means of the real representations of elliptic quaternion matrices, where $A_{s} \in \mathbb{H}_{\alpha}^{m \times n}, B_{s} \in \mathbb{H}_{\alpha}^{p \times q}, C \in \mathbb{H}_{\alpha}^{m \times q}$ and $s=1,2,3, \ldots, l$. 
Theorem 2.1. The elliptic quaternionic matrix equation given by (2.1) has a solution $X$ if and only if the real matrix equation

$$
\Phi_{A_{1}} Y \Phi_{B_{1}}+\cdots+\Phi_{A_{l}} Y \Phi_{B_{l}}=\Phi_{C}
$$

has a solution $Y \in \mathbb{R}^{4 n \times 4 p}$, in which case, if $Y \in \mathbb{R}^{4 n \times 4 p}$ is a solution of the real matrix equation (2.2), then the matrix

$$
X=\frac{1}{2-2 \alpha} E_{4 n} Y^{\prime}\left({ }^{1} \bar{E}_{4 p}\right)^{T}
$$

is a solution of (2.1) where

$$
Y^{\prime}=\frac{1}{4}\left(Y+R_{4 n} Y R_{4 p}^{-1}+S_{4 n} Y S_{4 p}^{-1}+T_{4 n} Y T_{4 p}^{-1}\right)
$$

and

$$
E_{4 t}=\left(\begin{array}{llll}
I_{t} & i I_{t} & j I_{t} & k I_{t}
\end{array}\right) \in \mathbb{H}_{\alpha}^{t \times 4 t}, t=n, p .
$$

Proof. Suppose that the real matrix

$$
Y=\left(\begin{array}{llll}
Y_{11} & Y_{12} & Y_{13} & Y_{14} \\
Y_{21} & Y_{22} & Y_{23} & Y_{24} \\
Y_{31} & Y_{32} & Y_{33} & Y_{34} \\
Y_{41} & Y_{42} & Y_{43} & Y_{44}
\end{array}\right), Y_{u v} \in \mathbb{R}^{n \times p}, u, v=1,2,3,4
$$

is a solution to the equation (2.2), then, we say that the matrix given in (2.3) is a solution to equation (2.1). According to Theorem 1.3, we get

$$
\begin{aligned}
& \Phi_{A_{s}}=R_{4 m}^{-1} \Phi_{A_{s}} R_{4 n}, \Phi_{B_{s}}=R_{4 p}^{-1} \Phi_{B_{s}} R_{4 q} \text { and } \Phi_{C}=R_{4 m}^{-1} \Phi_{C} R_{4 q} \\
& \Phi_{A_{s}}=S_{4 m}^{-1} \Phi_{A_{s}} S_{4 n}, \Phi_{B_{s}}=S_{4 p}^{-1} \Phi_{B_{s}} S_{4 q} \text { and } \Phi_{C}=S_{4 m}^{-1} \Phi_{C} S_{4 q} \\
& \Phi_{A_{s}}=T_{4 m}^{-1} \Phi_{A_{s}} T_{4 n}, \quad \Phi_{B_{s}}=T_{4 p}^{-1} \Phi_{B_{s}} T_{4 q} \text { and } \Phi_{C}=T_{4 m}^{-1} \Phi_{C} T_{4 q} .
\end{aligned}
$$

where $s=1,2,3, \ldots, l$. Substituting them into (2.2), respectively, and simplifying the corresponding equation, we have three equations as follows,

$$
\begin{aligned}
& \Phi_{A_{1}}\left(R_{4 n} Y R_{4 p}^{-1}\right) \Phi_{B_{1}}+\cdots+\Phi_{A_{l}}\left(R_{4 n} Y R_{4 p}^{-1}\right) \Phi_{B_{l}}=\left(\Phi_{C}\right), \\
& \Phi_{A_{1}}\left(S_{4 n} Y S_{4 p}^{-1}\right) \Phi_{B_{1}}+\cdots+\Phi_{A_{l}}\left(S_{4 n} Y S_{4 p}^{-1}\right) \Phi_{B_{l}}=\left(\Phi_{C}\right), \\
& \Phi_{A_{1}}\left(T_{4 n} Y T_{4 p}^{-1}\right) \Phi_{B_{1}}+\cdots+\Phi_{A_{l}}\left(T_{4 n} Y T_{4 p}^{-1}\right) \Phi_{B_{l}}=\left(\Phi_{C}\right) .
\end{aligned}
$$

This equation express that if $Y$ is a solution of the equation given by (2.2), then $R_{4 n} Y R_{4 p}^{-1}, S_{4 n} Y S_{4 p}^{-1}$ and $T_{4 n} Y T_{4 p}^{-1}$ are also solutions of the real matrix equation defined by (2.2). Thus the undermentioned real matrix:

$$
Y^{\prime}=\frac{1}{4}\left(Y+R_{4 n} Y R_{4 p}^{-1}+S_{4 n} Y S_{4 p}^{-1}+T_{4 n} Y T_{4 p}^{-1}\right)
$$

is a solution to (2.2). By substituting (2.5) in (2.7) and making necessary simplifications, it can easily be written by

$$
Y^{\prime}=\left(\begin{array}{cccc}
Z_{0} & \alpha Z_{1} & Z_{2} & \alpha Z_{3} \\
Z_{1} & Z_{0} & Z_{3} & Z_{2} \\
Z_{2} & \alpha Z_{3} & Z_{0} & \alpha Z_{1} \\
Z_{3} & Z_{2} & Z_{1} & Z_{0}
\end{array}\right)
$$

where 


$$
\begin{aligned}
& Z_{0}=\frac{1}{4}\left(Y_{11}+Y_{22}+Y_{33}+Y_{44}\right), Z_{1}=\frac{1}{4}\left(\frac{Y_{12}}{\alpha}+Y_{21}+\frac{Y_{34}}{\alpha}+Y_{43}\right), \\
& Z_{2}=\frac{1}{4}\left(Y_{13}+Y_{24}+Y_{31}+Y_{42}\right), Z_{3}=\frac{1}{4}\left(\frac{Y_{14}}{\alpha}+Y_{23}+\frac{Y_{32}}{\alpha}+Y_{41}\right) .
\end{aligned}
$$

Thus, we get $\Phi_{X}=Y^{\prime}$. From Theorem 1.3, we obtain

$$
X=\frac{1}{2-2 \alpha}\left(I_{n} i I_{n} j I_{n} k I_{n}\right) Y^{\prime}\left(\begin{array}{c}
I_{p} \\
-i I_{p} \\
j I_{p} \\
-k I_{p}
\end{array}\right)=Z_{0}+Z_{1} i+Z_{2} j+Z_{3} k
$$

Moreover, since $\Phi_{X}=Y^{\prime}$ the elliptic quaternionic matrix equation given in (2.1) has a solution if and only if the real matrix equation given in (2.2) has a solution.

\section{Numerical algorithm}

Considering the discussions in the previous section, now, we provide numerical Algorithm for solving general linear elliptic quaternionic matrix equation

$$
A_{1} X B_{1}+\cdots+A_{l} X B_{l}=C
$$

where $A_{s} \in \mathbb{H}_{\alpha}^{m \times n}, B_{s} \in \mathbb{H}_{\alpha}^{p \times q}, C \in \mathbb{H}_{\alpha}^{m \times q}$ and $s=1,2,3, \ldots, l$.

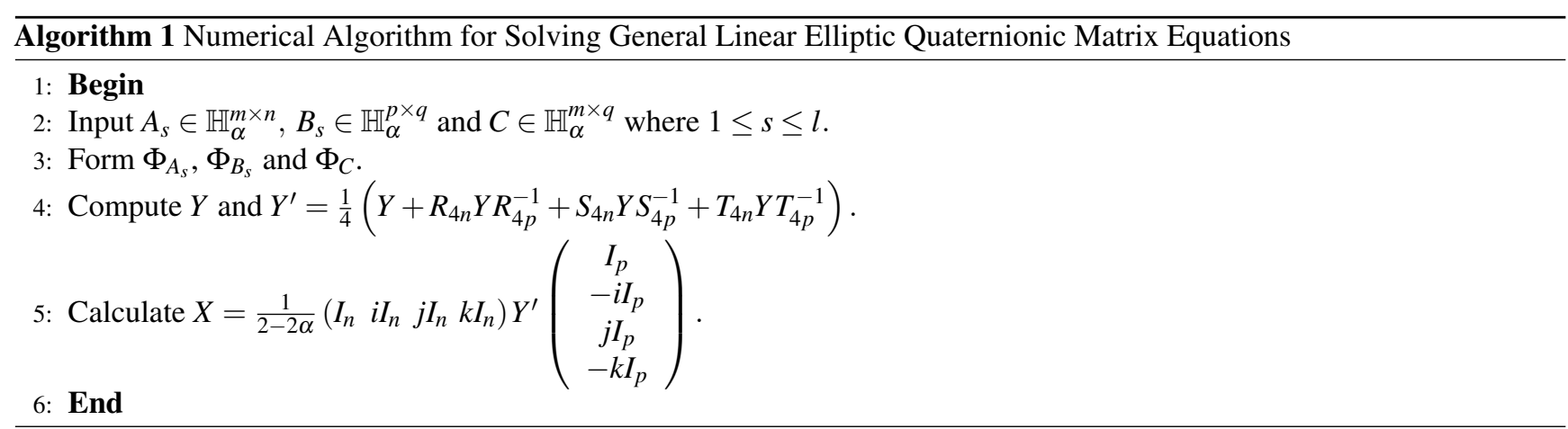

\section{Numerical examples}

For $l=2$, the special case of (2.1) is given by

$$
A_{1} X B_{1}+A_{2} X B_{2}=C
$$

where $A_{1}, A_{2} \in \mathbb{H}_{\alpha}^{m \times n}, B_{1}, B_{2} \in \mathbb{H}_{\alpha}^{p \times q}$ and $C \in \mathbb{H}_{\alpha}^{m \times q}$. If $B_{1}=I_{p}, A_{2}=-I_{n}, m=n, p=q$ are taken in (4.1), we have elliptic quaternionic Sylvester matrix equation $A X-X B=C$. Similarly, $A_{1}=I_{n}, B_{1}=I_{p}, m=n, p=q, A_{2}=-A$ and $B_{2}=B$ are taken in (4.1) we have elliptic quaternionic Kalman-Yakubovich matrix equation $X-A X B=C$.

In the literature, the equations $A X-X B=C$ and $X-A X B=C$ are known as the Sylvester matrix equation and the KalmanYakubovich matrix equation, respectively. These equations play an important role in control theory, signal processing, filtering, image restoration, decoupling techniques for ordinary and partial differential equations, and block-diagonalization of matrices, [14]-[18]. In this section, we obtain the solutions of the given elliptic quaternionic matrix equations $A X-X B=C$ and $X-A X B=C$ according to our Algorithm.

Note that all computations in the rest of the paper are performed on an Intel i7-3630QM@2.40 GHz/16GB computer using MATHEMATICA 9 software.

Let's take $\alpha=-2$ specifically to solve the elliptic quaternionic Kalman Yakubovich matrix equation

$$
X-\left(\begin{array}{cc}
1+k & i \\
j-k & 1-j
\end{array}\right) X\left(\begin{array}{cc}
j & 1+2 i \\
k & i+j
\end{array}\right)=\left(\begin{array}{cc}
3+i+3 j+k & 2+2 i+7 j+k \\
5+2 i-6 j+k & -7-2 i-2 j+8 k
\end{array}\right) .
$$


Real representation of given equation is

$$
Y-\left(\begin{array}{cccccccc}
1 & 0 & 0 & -2 & 0 & 0 & -2 & 0 \\
0 & 1 & 0 & 0 & 1 & -1 & 2 & 0 \\
0 & 1 & 1 & 0 & 1 & 0 & 0 & 0 \\
0 & 0 & 0 & 1 & -1 & 0 & 1 & -1 \\
0 & 0 & -2 & 0 & 1 & 0 & 0 & -2 \\
1 & -1 & 2 & 0 & 0 & 1 & 0 & 0 \\
1 & 0 & 0 & 0 & 0 & 1 & 1 & 0 \\
-1 & 0 & 1 & -1 & 0 & 0 & 0 & 1
\end{array}\right) Y\left(\begin{array}{cccccccc}
0 & 1 & 0 & -4 & 1 & 0 & 0 & 0 \\
0 & 0 & 0 & -2 & 0 & 1 & -2 & 0 \\
0 & 2 & 0 & 1 & 0 & 0 & 1 & 0 \\
0 & 1 & 0 & 0 & 1 & 0 & 0 & 1 \\
1 & 0 & 0 & 0 & 0 & 1 & 0 & -4 \\
0 & 1 & -2 & 0 & 0 & 0 & 0 & -2 \\
0 & 0 & 1 & 0 & 0 & 2 & 0 & 1 \\
1 & 0 & 0 & 1 & 0 & 1 & 0 & 0
\end{array}\right)
$$

If we solve this equation, we have

$$
Y=\left(\begin{array}{cccccccc}
1 & 0 & 2 & 0 & 0 & 1 & 0 & 0 \\
1 & 0 & 0 & 0 & 0 & 1 & -2 & -4 \\
-1 & 0 & 1 & 0 & 0 & 0 & 0 & 1 \\
0 & 0 & 1 & 0 & 1 & 2 & 0 & 1 \\
0 & 1 & 0 & 0 & 1 & 0 & 2 & 0 \\
0 & 1 & -2 & -4 & 1 & 0 & 0 & 0 \\
0 & 0 & 0 & 1 & -1 & 0 & 1 & 0 \\
1 & 2 & 0 & 1 & 0 & 0 & 1 & 0
\end{array}\right)
$$

Then

$$
\begin{aligned}
X & =\frac{1}{24}\left(\begin{array}{cccc}
I_{2} & i I_{2} & j I_{2} & k I_{2}
\end{array}\right)\left(Y+R_{8} Y R_{8}^{-1}+S_{8} Y S_{8}^{-1}+T_{8} Y T_{8}^{-1}\right)\left(\begin{array}{c}
I_{2} \\
-i I_{2} \\
j I_{2} \\
-k I_{2}
\end{array}\right) \\
& =\left(\begin{array}{cc}
1-i & j \\
1+k & j+2 k
\end{array}\right) .
\end{aligned}
$$

Similarly, let's take $\alpha=-5$ specifically to solve the elliptic quaternionic Sylvester matrix equation

$$
\left(\begin{array}{cc}
1+i & i+3 j+2 k \\
3 k & 2
\end{array}\right) X-X\left(\begin{array}{cc}
i & j+2 k \\
5+i & 2-3 j
\end{array}\right)=\left(\begin{array}{cc}
-46+13 i-19 j+k & -19+6 i-35 j+15 k \\
25-22 i-8 j+7 k & 48-6 i+21 k
\end{array}\right) .
$$

The solution of real representation of given elliptic quaternionic Sylvester matrix equation is

$$
Y=\left(\begin{array}{cccccccc}
1 & 2 & -5 & 0 & 1 & 0 & 0 & 0 \\
0 & 0 & 0 & -25 & 1 & 3 & -20 & 0 \\
1 & 0 & 1 & 2 & 0 & 0 & 1 & 0 \\
0 & 5 & 0 & 0 & 4 & 0 & 1 & 3 \\
1 & 0 & 0 & 0 & 1 & 2 & -5 & 0 \\
1 & 3 & -20 & 0 & 0 & 0 & 0 & -25 \\
0 & 0 & 1 & 0 & 1 & 0 & 1 & 2 \\
4 & 0 & 1 & 3 & 0 & 5 & 0 & 0
\end{array}\right)
$$

Thus, we get

$$
\begin{aligned}
& X=\frac{1}{24}\left(\begin{array}{cccc}
I_{2} & i I_{2} & j I_{2} & k I_{2}
\end{array}\right)\left(Y+R_{8} Y R_{8}^{-1}+S_{8} Y S_{8}^{-1}+T_{8} Y T_{8}^{-1}\right)\left(\begin{array}{c}
I_{2} \\
-i I_{2} \\
j I_{2} \\
-k I_{2}
\end{array}\right) \\
& =\left(\begin{array}{cc}
1+i+j & 2 \\
j+4 k & 5 i+3 j
\end{array}\right) .
\end{aligned}
$$




\section{Conclusion}

In this study, we established the solution of general linear elliptic quaternionic matrix equations with the help of the real representation of elliptic quaternion matrices and expressed an Algorithm for the solutions of these equations. In addition, we investigated solutions of elliptic quaternionic Sylvester and Kalman Yakubovich matrix equations, which are essential applications in various areas of science. Actually, general linear matrix equations over the complex field form a special class of general linear elliptic quaternionic matrix equations. Thus, the obtained results extend, generalize and complement the scope of general linear matrix equations known in the literature.

\section{Acknowledgements}

The authors would like to express their sincere thanks to the editor and the anonymous reviewers for their helpful comments and suggestions.

\section{Funding}

There is no funding for this work.

\section{Availability of data and materials}

Not applicable.

\section{Competing interests}

The authors declare that they have no competing interests.

\section{Author's contributions}

All authors contributed equally to the writing of this paper. All authors read and approved the final manuscript.

\section{References}

[1] W. R. Hamilton, Lectures on Quaternions, Hodges and Smith, Dublin, 1853.

[2] Y. Tian, Universal factorization equalities for quaternion matrices and their applications, Math. J. Okoyama Univ., 41 (1999), $45-62$.

[3] S. L. Adler, Quaternionic Quantum Mechanic and Quantum Fields, Oxford U. P., New York, 1994.

[4] C. K. C. Jack, Quaternion kinematic and dynamic differential equations, IEEE Trans Robotics and Automation, 8 (1992), 53-64

[5] S. Salamon, Differential geometry of quaternionic manifolds, Ann. Sci. Ec. Norm. Sup. Paris, 19 (1986), 31-54.

[6] S. C. Pei, C. M. Cheng, Quaternion matrix singular value decomposition and its applications for color image processing, Int. Conf. Image Processing, 1 (2003), 805-808.

[7] C. Segre, The real representations of complex elements and extension to bicomplex systems, Math. Ann., 40 (1892), $413-467$.

[8] F. Catoni, R. Cannata, P. Zampetti, An introduction to commutative quaternions, Adv. Appl. Clifford Algebras, 16 (2006), 1-28.

[9] S. C. Pei, J. H. Chang, J. J. Ding, Commutative reduced biquaternions and their Fourier transform for signal and image processing applications, IEEE Transactions on Signal Processing, 52 (2004), 2012-2031.

[10] S. C. Pei, J. H. Chang, J. J. Ding, M. Y. Chen, Eigenvalues and singular value decompositions of reduced biquaternion matrices, IEEE Trans. Circ. Syst. I., 55 (2008), 2673-2685.

[11] T. Isokawa, H. Nishimura, N. Matsui, Commutative quaternion and multistate Hopfield neural networks, In Proc. Int. Joint Conf. Neural Netw., (2010),

[12] H. H. Kosal, On the Commutative quaternion matrices, Ph. D. Thesis, Sakarya University, 2016.

[13] H. H. Kosal, An Algorithm for solutions to the elliptic quaternion matrix equation AX =B, CPOST., 1(1) (2018), 36-40.

[14] A. Jameson, Solution of the equation $a x+x b=c$ by inversion of an $m \times m$ or $n \times n$ matrix, SIAM J. Appl. Math., 16(5)(1968), 1020-1023.

[15] E. Souza, S. P. Bhattacharyya, Controllability, observability and the solution of $a x-x b=c$, Linear Algebra Appl., 39(1981), $167-188$.

[16] M. Dehghan, M. Hajarian, Efficient iterative method for solving the second-order Sylvester matrix equation EV $F^{2}-A V F-C V=B W$, IET Contr. Theory Appl., 3(10)(2009), 1401-1408.

[17] C. Song, G. Chen, On solutions of matrix equations $X F-A X=C$ and $X F-A \tilde{X}=C$ over quaternion field, J. Appl. Math. Comput., 37(1-2)(2011), 57-68.

[18] X. Zhang, A system of generalized Sylvester quaternion matrix equations and its applications, Appl. Math. Comput., 273 (2016), $74-81$. 